\title{
Ruptured anterior communicating artery aneurysm presenting with monocular blindness
}

Sir,

Subarchnoid hemorrhage (SAH) is the common 
presenting feature of anterior communicating artery (Acoma) aneurysms. The usual locations of the aneurysms presenting with visual deficits are cavernous, carotid-ophthalmic, supraclinoid, paraclinoid, posterior communicating. ${ }^{[1]}$ Only a few cases of Acoma aneurysms presenting with visual deficits have been reported in the literature. ${ }^{[1-3]}$

A 45-year-old female patient presented in emergency with sudden onset headache, vomiting, and right eye vision loss. Vision loss was sudden and complete. On examination, she was conscious and well oriented. Her vision and pupil on left side was normal, whereas on right side there was no perception of light; right pupil was dilated and nonreacting. She also had neck stiffness. Cranial computed tomography (CT) scan showed diffuse SAH in anterior interhemispheric fissure, suprasellar cistern, and bilateral sylvian fissures [Figure 1a]. CT angiography showed a large pedunculated Acoma aneurysm $75 \times 45 \times 35 \mathrm{~mm}^{3}$ with fundus directed anteroinferiorly, towards right side with $3 \mathrm{~mm}$ long and narrow neck ( $2 \mathrm{~mm})$; and right A1 segment was attenuated [Figure 1b]. She was subjected to pterional craniotomy and clipping of Acoma aneurysm. Intraoperatively, the Acoma aneurysm was found to be projecting into right optic nerve. There was dense adhesion between the aneurysm sac and the optic nerve with evidence of hemorrhage into the nerve. Gently aneurysm was dissected and was clipped with an asculap permanent $6.5 \mathrm{~mm}$ curved clip. There was no intraoperative rupture and no temporary clipping was used. Postoperative period was uneventful. Vision in the right eye did not improve. At one year follow-up, patient was fully conscious and oriented, but the vision in the right eye had still not improved.

\section{Ruptured Acoma aneurysms generally present with}

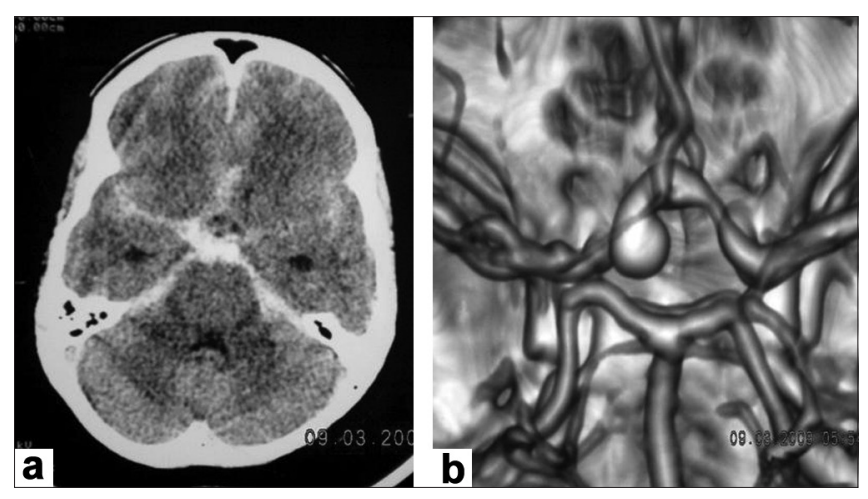

Figure 1: (a) Noncontrast computed tomography scan showing diffuse subarchnoid hemorrhage in interhemishperic, suprasellar, and bilateral sylvian cistern; (b) Computed tomography angiography showing a large coma aneurysm with fundus directed anteroinferiorly and towards right side with attenuated right $A 1$ segment
$\mathrm{SAH}$ and associated focal neurological deficits. The various causes of visual deficits include: Compression of the optic apparatus, ${ }^{[1]}$ leaking of aneurysm inside the optic nerve or chiasma, ${ }^{[2]}$ perianeurysmal inflammatory changes, ${ }^{[3]}$ thromboembolism, and vitreous hemorrhage (terson's syndrome). ${ }^{[4]}$ Only a few ruptured Acoma aneurysms with visual deficits such as monocular blindness have been reported till date. ${ }^{[1-3]}$ In most of the patients visual deficits were related to chiasmal field defects because of the proximity of Acoma complex to the optic chiasma rather than optic nerve. Isolated involvement of one optic nerve resulting in monocular blindness has not been reported before. In the present case, monocular blindness was probably due to the direction of the growth of fundus of large Acoma aneurysm and compression of the optic nerve and also due to the hemorrhage into the optic nerve., Distortion or traction and indirect pressure on the optic nerves at the margins of the optic foramina also play an important role. ${ }^{[3]}$ Other factor is interference with the blood supply of the optic nerve or chiasma either by occlusion or distortion of the perforating arteries arising from anterior cerebral-communicating complex. In large Acoma aneurysms, the intraaneurysmal thrombosis may occlude the origins of the branch vessels supplying the optic nerves and chiasma and thus causing ischemia. Sometimes, if the optic nerve is adherent to the fundus of the Acoma aneurysm there may also be damage from hemorrhage into the neural substance. ${ }^{[3]}$ This was the most likely cause of monocular blindness in our case. This was also confirmed intraoperatively as fundus of Acoma aneurysm was compressing the optic nerve with hemorrhagic staining of the optic nerve. Patients with unruptured aneurysms may present only with progressive visual loss, due to the compression of optic pathway by enlarging aneurysmal sac or other causes. Thus neurologists, neurosurgeons, and ophthalmologists should have high index of suspicion and should consider unruptured Acoma aneurysm as one of the differential diagnosis for monocular blindness.

\section{Alok A. Umredkar, Navneet Singla, Sunil K. Gupta}

Department of Neurosurgery, Postgraduate Institute of Medical Education and Research, Chandigarh, India. E-mail: alokumr@yahoo.co.in

\section{References}

DOI: $10.4103 / 0028-3886.59498$

1. Bakker SL, Hasan D, Bijvoet HW. Compression of the visual pathway by anterior cerebral artery aneurysm. Acta Neurol Scand 
1999;99:204-7.

2. Cullen JF, Haining WM, Crombie AL. Cerebral aneurysms presenting with visual field defects. Br J Ophthalmol 1966;50:251-6.

3. Peiris JB, Ross Russell RW. Giant aneurysms of the carotid system presenting as visual field defect. J Neurol Neurosurg Psychiatry 1980;43:1053-64.

4. Roux FX, Panthier JN, Tanghe YM, Gallina P, Oswald AM, Mérienne L, et al. Terson's syndrome and intraocular complications in meningeal hemorrhages (26 cases). Neurochirurgie 1991;37:106-10.

Accepted on 02-07-2009 